\title{
AIRBORNE LIDAR POINT CLOUD CLASSIFICATION BASED ON MULTILEVEL POINT CLUSTER FEATURES
}

\author{
Yuan $\mathrm{Gao}^{1, *}$, Mingchu $\mathrm{Li}^{1}$ \\ 1.School of Software Technology, Dalian University of Technology, Dalian 116620,China; 937018401@qq.com
}

KEY WORDS: Airborne LiDAR, Hierarchical point cluster, Sparse coding, LDA, Hierarchical feature, Point clouds classifications

\begin{abstract}
:
Airborne Light Detection And Ranging (LiDAR) has become an important means for efficient and high-precision acquisition of 3D spatial data of large scenes. It has important application value in digital cities and location-based services. The classification and identification of point cloud is the basis of its application, and it is also a hot and difficult problem in the field of geographic information science.The difficulty of LiDAR point cloud classification in large-scale urban scenes is: On the one hand, the urban scene LiDAR point cloud contains rich and complex features, many types of features, different shapes, complex structures, and mutual occlusion, resulting in large data loss; On the other hand, the LiDAR scanner is far away from the urban features, and is like a car, a pedestrian, etc., which is in motion during the scanning process, which causes a certain degree of data noise of the point cloud and uneven density of the point cloud.

Aiming at the characteristics of LiDAR point cloud in urban scene. The main work of this paper implements a method based on the saliency dictionary and Latent Dirichlet Allocation (LDA) model for LiDAR point cloud classification. The method uses the tag information of the training data and the tag source of each dictionary item to construct a significant dictionary learning model in sparse coding to expresses the feature of the point set more accurately.And it also uses the multi-path AdaBoost classifier to perform the features of the multi-level point set. The classification of point clouds is realized based on the supervised method. The experimental results show that the feature set extracted by the method combined with the multi-path classifier can significantly improve the cloud classification accuracy of complex city market attractions.
\end{abstract}

\footnotetext{
* Yuan Gao - E-mail:937018401@qq.com.
} 


\section{INTRODUCTION}

The difficulty of laser radar point cloud classification in largescale urban scenes lies in: on the one hand, urban scenes have many types of objects, different shapes, complex structures, mutual occlusion, and like cars, pedestrians, etc. are in motion during scanning. It causes a certain degree of stretching and deformation of the point cloud. On the other hand, the LiDAR scanner is far away from the urban ground object, which reduces the accuracy of the point cloud and brings higher data noise.

LiDAR point cloud classification methods based on point sets and contexts need to extract and construct single point or point set features [1]. Single point features mainly include average points, average normal vectors [2], etc. The calculation of feature based on single point is relatively simple, and can express the difference of characteristics of each point differently from other points, but this kind of feature ignores the feature similarity and interrelationship between adjacent points, and the density of point clouds. Changes and noise are sensitive and can easily lead to the misclassification of different types of morphologically similar areas in complex urban scenes. The feature of the point set inherits the advantages of the single point feature, and considers the correlation between adjacent points, which is not sensitive to the change of the point density, but needs to determine the size of the support area of each point reasonably. This paper mainly classifies point clouds by constructing multi-

level point sets and describing the characteristics of point sets in each level.

This paper proposes a new framework to extract shape features from features in urban LiDAR point cloud scenes, and then uses the multipath AdaBoost classifier to classify the ground objects.

The frame is particularly suitable for identifying objects with relatively small shapes, such as cars and pedestrians. First, based on the natural exponential function threshold algorithm, the input LiDAR point cloud that removes the ground point is segmented into Nature Exponential-based Hierarchical Point Clusters (NEHPCs); then, the Sparse Coding and Latent Dirichlet Allocation (SCLDA) is used to construct the shape features of the point set based on the single point feature; finally, the AdaBoost classifier is used to distinguish ground objects from the LiDAR point cloud, and the LiDAR point cloud of different urban scenes is used to test the method.

The main contributions of this section are as follows:

1) Considering the difference in the spatial extent of the object entities, we propose a method based on the natural exponential function to construct a multi-level point set, that is, use the natural exponential form to control the segmentation thresholds of each level in the point cloud, the point cloud is then segmented using graph cuts [3] and normalized cuts [4]. Since the constructed point set is multi-level, the features extracted from the multi-level point set are more robust than the pointbased features, and they can adapt to the change of the point cloud density.

2) Use SCLDA to extract the shape features of each point set in NEHPCs. Since SCLDA combines the advantages of sparse coding and LDA, LDA can extract features of the same length from a set of points with different numbers of points. Sparse coding can identify the correlation between different features, eliminate redundant expression, and retain significant features. SCLDA can better express the feature of point set, and at the same time, further improve the degree of discrimination of the set of points to be discriminated by means of the inheritance of different sets of point sets.

\section{RELATED WORK}

Biosca et al. [5] used unsupervised fuzzy clustering to planarly segment the point cloud, which can also be extended to the recognition of surface morphology of non-planar objects. Barnea and Filin [6] converted the point cloud into a depth map, and then used the image, and used the mean-shift algorithm [7] to separate the depth map and the image independently. Finally, they combined the segmentation results to obtain good clustering. Then, based on the previous work [7], Barnea improved the segmentation algorithm of depth map and image [8]. In order to get better segmentation boundary and segmentation region, an iterative segmentation method was designed, but the method cannot achieve the purpose of segmentation of the target object. Rusu et al. [9] mainly classify indoor LiDAR point clouds, including kitchen scenes. By dividing the indoor point cloud meaningfully, the main segmentation unit is a meaningful entity, by resampling and removing outliers. To preserve the gap between the cabinet doors, this method can only be segmented for a single object, and at the same time, it needs to have prior knowledge of the features in the scene. Yokoyama et al. [10] extracted rod-shaped features in the on-board LiDAR cloud. First, in order to highlight the rod-shaped features and the planar features, the point cloud is contracted by Laplacian smoothing, and then the point cloud is formed into a rod-shaped, planar, and mixed ground by clustering, finally, they identified the rod-like features through some combination rules.

Multi-level neighborhoods can be implemented by setting the multi-level neighborhood size [11] or adaptively determining the neighborhood size based on the scale parameter [12]. In addition to multi-level neighborhoods, multi-scale features based on multi-level segmentation have also been studied by many scholars. Wang et al. [13] used a multi-scale multi-level framework to handle Terrestrial Laser Scanning (TLS) point clouds in complex urban scenes. Within this framework, the point cloud is first resampled into point clouds of different scales. The point clouds resampled at each scale are aggregated into multi-level point sets, and the point set features at each level of each scale are expressed by means of LDA. Then, each point set is classified by the classifier to be classified into the respective categories. Another method based on multi-level segmentation is used in image classification [14]. This method divides the image into multi-level superpixels, and then aggregates the local neighborhood points with similar color and texture features into one class. Brodu and Lague [15] used multi-scale local features to classify TLS point clouds. Because of the combination of features at different scales, this method has better classification than single-scale features. The classification of TLS point clouds with missing partial data has certainly robust. Pauly et al. [16] proposed a multi-scale classification framework for discrete surface analysis and multiscale feature extraction in a multiscale framework. Xiong et al. [17] subdivided point cloud data from coarse to subdivided into multiple levels. These levels include pointbased and region-based hierarchies. In this multi-level structure, the previous level of discrimination is used. At a level, semantic features are formed, and point cloud classification is performed by means of statistics and associated information. Xu et al. [18] used three levels of point cloud classification, namely single point level, plane segmentation level and level segmented by 
mean-shift method [7]. The method extracts features at these three levels for segmentation. The contextual and shape features of the point cloud are determined from different levels. Based on spectral information, geometry and topological features, the literature [19] proposes a rule-based multi-level semantic classification framework.

The object entities in the LiDAR point cloud scene are rich and complex, and a discriminative feature that is insensitive to point density is needed to describe the objects in the point cloud. At the same time, this feature needs to have scale invariance. There is a certain difference in the point density of objects in the point cloud, which may result in different point cloud distributions of the same species, and different types of features have the same point cloud distribution locally. Therefore, the feature of expressing a feature needs to have a high degree of discrimination. However, local features in a single range are difficult to identify the object entities better, and different local ranges are used to identify the ground objects, which can get better recognition results. Therefore, we propose a multi-level point cloud classification framework, which can effectively obtain the shape features of objects from complex airborne laser point cloud scenes. Each level of point set trains the respective classifier. By combining the discriminant results of the different levels of the point set to be identified, it is possible to determine the category of the set of points to be identified from different spatial ranges, and improve the distinguishability of the feature of the point set. Further, a more accurate point set discrimination result is achieved. In this way, the set of points to be identified can be jointly classified by using different levels of recognition results. The multi-level architecture can effectively improve the classification accuracy of object point clouds, especially the classification accuracy of small objects.

\section{METHOD}

\subsection{Build a multi-level point set}

The extraction of single-point features requires a certain support area, and the size of the support area affects the quality of the point features, especially in areas with complex scenes. In order to overcome the influence of single point features on classification instability, we cluster point clouds into point sets according to certain rules, and use point sets to judge the category attribution of single points. Compared with singlepoint feature classification, the point set-based method is more stable and efficient, because it contains and integrates more and more significant feature information.

This paper proposes a point threshold control method based on natural index change to construct a multi-level point set. The multi-level point set generated by this method has certain variability, and different levels of point sets can cover different spatial ranges. The point cloud is segmented by the method of this paper. Entities (such as buildings and cars) with large spatial differences in the point cloud can be synchronized and fully segmented, especially small objects that are difficult to segment. Different levels of point sets show good variability, and different range of point sets can provide more abundant spatial features, which is helpful for the identification of target point sets. Building a multi-level point set includes the following steps[20]:

(1) Remove ground points and isolated points. Because this paper mainly studies an efficient method based on point set entity shape feature, and classifies and recognizes different entities based on shape features, we need to remove ground points.

(2) Construct a graph structure of the point cloud. After removing the ground point, most of the features in the point cloud are isolated. To segment the point cloud, the point cloud after removing the ground point needs to be organized in a certain way.

Perform a preliminary segmentation of the point cloud

(3) using the graph cut method. Because some areas in a point cloud scene are more complex, such as some features have some aggregation or occlusion, the connected components of the area may contain multiple feature entities, so we need to further segment the connected components. Graph cuts can minimize the similarity between categories, and the intraclass similarity is the largest. By disconnecting the edges with the least weight among the connected components, the connections inside each feature are the closest.

(4) After the above segmentation, although some aggregated point sets are segmented to some extent, there are still point sets containing multiple features in the point set, which is determined by the complexity of the scene. In order to obtain a higher-resolution point set feature, a point set can only contain one feature or a part of the feature, and the point distribution in each point set is relatively consistent. Since the normalized cuts balance the relationship between the points of the point sets and the points inside the point sets, not only the weight of the edge between the points is minimized, but also the spatial extent between the points is not much different. Therefore, we use the normalized cuts method to divide the point cloud into multilevel point sets, which can effectively divide the point set into point sets with similar distribution, and at the same time separate the points with dissimilar distribution.

\subsection{Multi-level point set features construction}

Different levels of point sets contain different numbers of points. In order to better express the characteristics of point sets, inspired by the BoW method, we introduce sparse coding to describe the characteristics of point sets. Sparse coding has obvious advantages in dictionary extraction and feature representation. It has the following basic assumptions: input data can be represented by a linear combination of words in an overcomplete dictionary. Among them, the dictionary can be obtained through point-based feature training. First, we define a point set as a document, and all the point sets make up a set of documents. A dictionary obtained by sparse coding is defined as a dictionary of LDA. Each point-based feature in the point set is used as the basic unit, and the features of the point are sparsely expressed using sparse coding. In each point set, the frequency at which each word appears is calculated to generate a word frequency vector having a length of $\mathrm{N}$ (where $\mathrm{N}$ represents the number of words in the dictionary). The SCLDA model is trained through point-based features, which include the following steps:

(1) Point feature normalization

All point feature matrices $\mathrm{F}$ are normalized by column using equation (1). After all the columns are normalized, they are then trained in the dictionary and sparsely expressed.

$$
\boldsymbol{n}=\frac{f-\min }{\max -\min }
$$

where, $\mathrm{n}$ represents the normalized value of the sub-items in the feature vector, f represents the value of the current sub-item, max represents the maximum value in each column of $F$, and min represents the minimum value in each column of $F$.

(2) Construct a sparse coding model based on point features 
Let all point features be normalized to $X=\left[x_{1}, x_{2}, \ldots, x_{q}\right]^{t}$, where $\mathrm{q}$ is the number of point features. The sparse coding model for constructing the point feature $\mathrm{X}$, the dictionary $\mathrm{V}$, and the sparse expression $\mathrm{U}$ is as shown in the equation (2).

$$
\begin{gathered}
\langle V, U\rangle=\operatorname{argmin}\left(\|X-V U\|_{2}^{2}+\lambda|U|\right) \\
\text { Subject to }\left\|W_{k}\right\| \leq 1, \quad \forall k=1,2, \cdots, N,
\end{gathered}
$$

\section{(3) Training of the dictionary}

The training of the dictionary $\mathrm{V}$ is obtained by $\mathrm{U}$ and $\mathrm{V}$ iterative solution. When $\mathrm{V}$ is fixed, $\mathrm{U}$ is obtained by the Feature Sign Method; when $\mathrm{U}$ is fixed, $\mathrm{V}$ is solved by Lagrange Dual Method. Through such several iterations, when the objective function converges, the optimal dictionary $\mathrm{V}$ is obtained.

(4) Sparse expression

After the dictionary $\mathrm{V}$ is optimized, the sparse expression of the input point feature $\mathrm{X}$ is calculated, and the feature signature method is used to solve the sparse representation code $U$ based on the point feature.

(5) Extracting NEHPC features based on SCLDA

Through the above steps, the sparse expression result $U$ of each point feature is obtained, and the frequency of occurrence of each word in each point set needs to be counted, and the frequency at which each word appears forms a word frequency vector of $\mathrm{N}$-dimensional ( $\mathrm{N}$ represents the number of words in the dictionary).

The LDA model is established as follows:

$$
p(\boldsymbol{V} \mid \boldsymbol{\alpha}, \boldsymbol{\beta})=\frac{\Gamma\left(\sum_{i} \alpha_{i}\right)}{\prod_{i}\left(\Gamma\left(\alpha_{i}\right)\right)} \int\left(\prod_{i=1}^{k} \theta_{i}^{\alpha_{i}-1}\right)\left(\prod_{n=1}^{N} \sum_{z_{n}} p\left(z_{n} \mid \theta\right) p\left(\boldsymbol{w}_{n} \mid z_{n}, \boldsymbol{\beta}\right)\right) d \theta
$$

\subsection{Point cloud classification based on discriminative inheritance}

a specific object category. In the generalization phase, the most elaborate level of point set contains only one object or part of the object. Other levels of point sets may contain one or more feature entities, so we only mark the most fine-level point set. A point set and a set of points above it contain SCLDA features of different spatial extents. Therefore, the unknown point set achieves the classification purpose by inheriting the category discrimination result of the upper hierarchical point set. The probability that a point set is marked as $l_{i}$ is:

$$
\boldsymbol{P}_{n}^{j}\left(l_{i}\right)=\prod_{m=1}^{n} \boldsymbol{P}^{m, \text { num }}\left(l_{i}, \boldsymbol{F}_{S L}\right)
$$

where, $\mathrm{n}$ represents the total number of levels of the multi-level point set, $p_{n}^{j}$ represents the probability that the $\mathrm{j}$-th point set belongs to the category li, and $p_{n}^{\text {m,num }}$ represents the m-th point set attribution in the num-level point set belongs to the category li. Finally, the largest one of the probabilities marked as each class is taken as the tag recognition result of the point set at the finest level.

\section{EXPERIMENTAL RESULTS AND ANALYSIS}

The computer environment in which this algorithm runs is: 2.71 $\mathrm{GHz}$ Intel(R) Core(TM) i5-7300U CPU, 8GB RAM. The system takes about 35 minutes to learn the SCLDA model and the AdaBoost classifier. The identification of point clouds in scenes I, II and III takes approximately $28.5 \mathrm{~min}, 17.6 \mathrm{~min}$ and $10.8 \mathrm{~min}$. Feature extraction and sparse expression take about $60 \%$ of the total time during program execution. However, most
In order to test the multi-level point set features, we use the AdaBoost classifier to train the multi-level point set features based on the SCLDA model, and test the set of identified points, and classify the points into different categories. First, the training data is clustered into multi-level point sets, and then the multi-level point set features based on the SCLDA model are extracted from the multi-level points. After the multi-level point set feature based on the SCLDA model is constructed, the onevs-all strategy is used to obtain the AdaBoost classifier of each category through training. Assume that the ground objects are divided into three categories: buildings, trees and cars. The training data is divided into n-level point sets. 3n AdaBoost classifiers need to be trained. In the training process, SCLDA model parameters and AdaBoost classifiers are obtained in turn. The trained classifier is then used in the identification of unmarked point clouds.

This paper proposes a method to distinguish unmarked points from a coarser level to a fine level. First, the probability $P_{\text {num }}$ of the point set class attribution is designed. The mathematical expression of the probability $P_{\text {num }}\left(l_{i}, F^{S L}\right)$ that marks the point set as the label $l_{i}$ is:

$$
\boldsymbol{P}_{\text {num }}\left(l_{i}, \boldsymbol{F}^{S L}\right)=\frac{\exp \left(H_{\text {num }}\left(l_{i}, \boldsymbol{F}^{S L}\right)\right)}{\sum_{i} \exp \left(H_{\text {num }}\left(l_{i}, \boldsymbol{F}^{S L}\right)\right)}
$$

where, $F^{S L}$ is a feature obtained by each point set based on the SCLDA model, num is an integer $(1 \leq$ num $\leq \mathrm{n})$, and $H_{\text {num }}\left(l_{i}, F^{S L}\right)$ is a probability that the AdaBoost classifier marks the set of points to be marked as the category $l_{i}$.

Next, the unknown point set is marked according to the discriminative inheritance from the coarse to the fine level. During the training process, the training data is manually marked by manual visual interpretation, and each point set contains only

of the steps are parallelizable. Therefore, parallel strategies can be used to reduce time overhead.

In order to verify the method of this paper and compare it with other methods, we use three other methods to compare with the method. The basic characteristics of these four methods are shown in Table 1. The first method (method I) uses BoW and LDA to express the characteristics of the point set based on the multi-level point set, and uses the AdaBoost classifier to classify the unknown point cloud through the k-means method [21]-[22]. Training is performed to obtain a dictionary, and each point-based feature is expressed by vector quantization. The second method (method II) is classified by AdaBoost classifier on the basis of point features. This method does not divide the point cloud into point sets, and does not involve multi-level structure. It is classified and identified by single point as the basic unit. The third method (method III) is the method described in [2]. In this method, each feature is described by geometric, multi-echo strength, statistics, etc., and then the JointBoost method is used to select features and evaluate their correlation. However, the method does not use context information (such as intensity information, etc.) to identify point cloud, we only use the spatial correlation features based on multi-level point sets to identify the ground objects, and the point changes between the multi-level point sets are exponential. Precision and recall can be used to assess the quality of the classification [13]. The precision is the proportion of the retrieved instance in the retrieved instance; the recall rate is the proportion of the retrieved instance in the related instance to the related instance. High precision means that the algorithm can get more relevant results, and the high recall rate means that the algorithm can get most of the relevant results. Table 2 lists the 
accuracy, recall, and accuracy of the four methods in the learning phase. It can be seen from Table 2 that the accuracy and recall rate of the classification results obtained by the method is the highest. The precision/recall of this method is significantly higher than other methods. Compared with the other three methods, this paper is based on SCLDA. The multilevel point set feature of the model can better describe the training data and effectively distinguish the ground objects.

Table 1 MAIN CHARACTERISTICS OF METHODS

\begin{tabular}{c|c|c|c|c|c}
\hline Methods & $\begin{array}{c}\text { Feature } \\
\text { representation }\end{array}$ & $\begin{array}{c}\text { Vocabulary } \\
\text { extraction method }\end{array}$ & $\begin{array}{c}\text { Vocabulary } \\
\text { representation }\end{array}$ & $\begin{array}{c}\text { Cluster- } \\
\text { based }\end{array}$ & $\begin{array}{c}\text { Hierarchical point- } \\
\text { cluster sets }\end{array}$ \\
\hline Our Method & $\begin{array}{c}\text { SCLDA of point- } \\
\text { cluster sets }\end{array}$ & $\begin{array}{c}\text { Lagrange dual } \\
\text { method }\end{array}$ & $\begin{array}{c}\text { Feature sign } \\
\text { method }\end{array}$ & Yes & Exponential \\
\hline Method I & $\begin{array}{c}\text { LDA of point- } \\
\text { cluster sets }\end{array}$ & k-means method & $\begin{array}{c}\text { Vector } \\
\text { quantization }\end{array}$ & Yes & Exponential \\
\hline Method II & Point-based & No vocabulary & No vocabulary & No & No \\
\hline Method III & Point-based & No vocabulary & No vocabulary & No & No \\
\hline
\end{tabular}

Table 2 PRECISION/RECALL(\%) OF DIFFERENT METHODS

\begin{tabular}{c|c|c|c|c|c|c|c|c}
\hline & \multicolumn{2}{|c|}{ Our Method } & \multicolumn{2}{c|}{ Method I } & \multicolumn{2}{c|}{ Method II } & \multicolumn{2}{c}{ Method III } \\
\hline Scene & precision & recall & precision & recall & precision & recall & precision & recall \\
\hline I & 98.3 & 97.9 & 94.9 & 95.1 & 90.2 & 94.6 & 95.5 & 96.8 \\
\hline II & 91.5 & 96.7 & 92.5 & 96.6 & 79.5 & 92.8 & 90.4 & 93.5 \\
\hline III & 93.8 & 86.9 & 78.3 & 84.7 & 80.1 & 59.4 & 84.7 & 62.6 \\
\hline
\end{tabular}


The International Archives of the Photogrammetry, Remote Sensing and Spatial Information Sciences, Volume XLII-3/W10, 2020

International Conference on Geomatics in the Big Data Era (ICGBD), 15-17 November 2019, Guilin, Guangxi, China

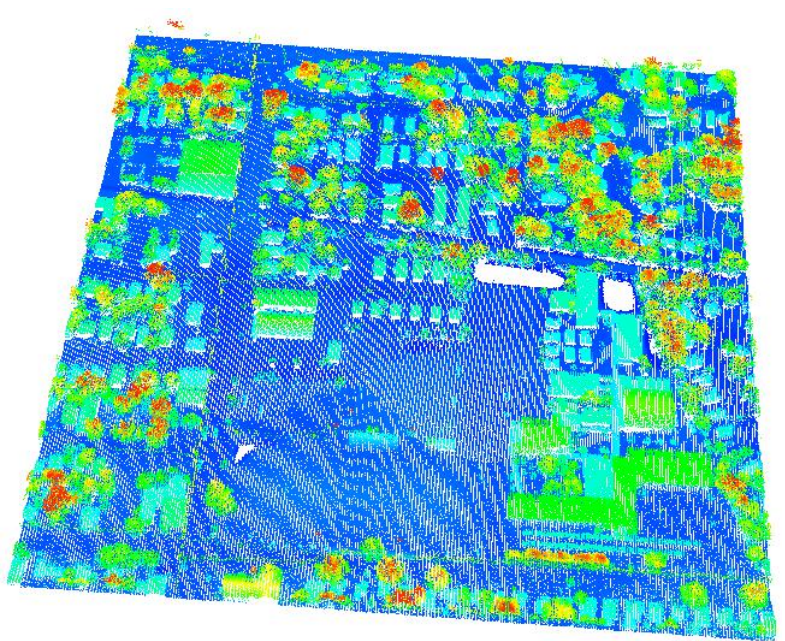

(a)raw data

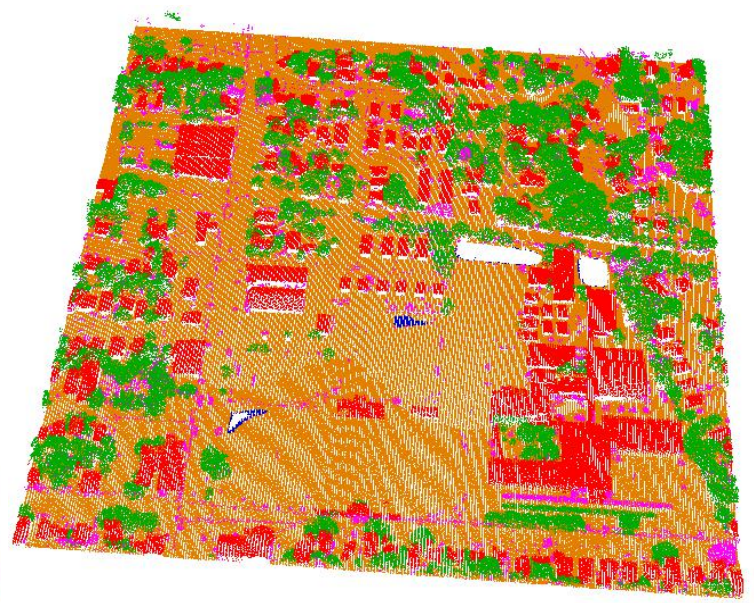

(b)classification results

Figure 1. Results of scene I

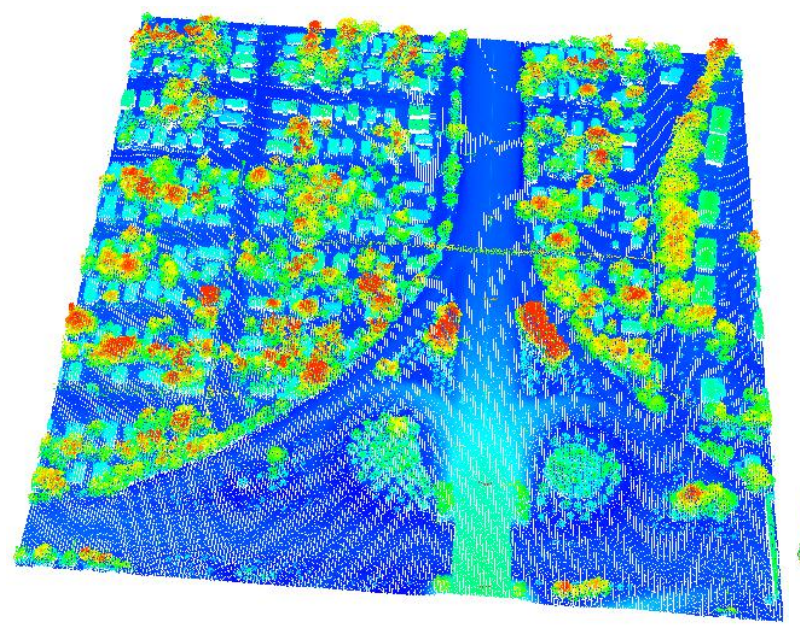

(a)raw data

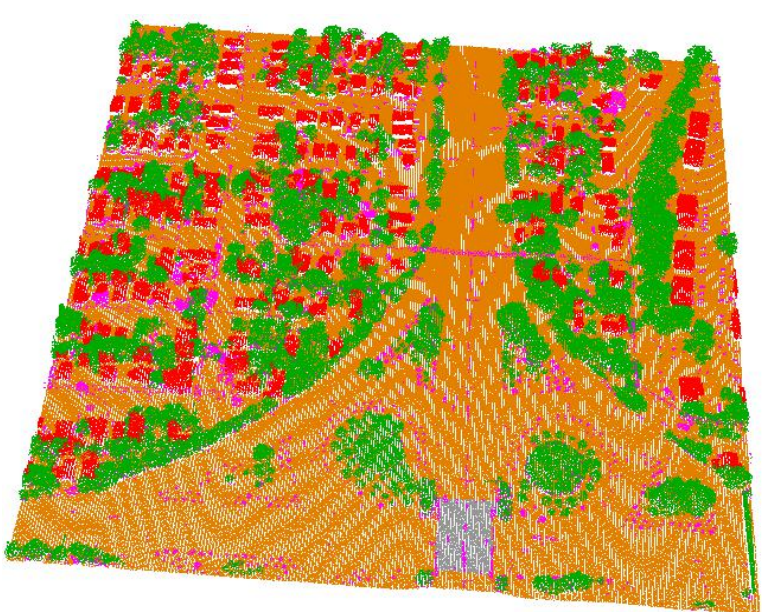

(b)classification results

Figure 2. Results of scene II

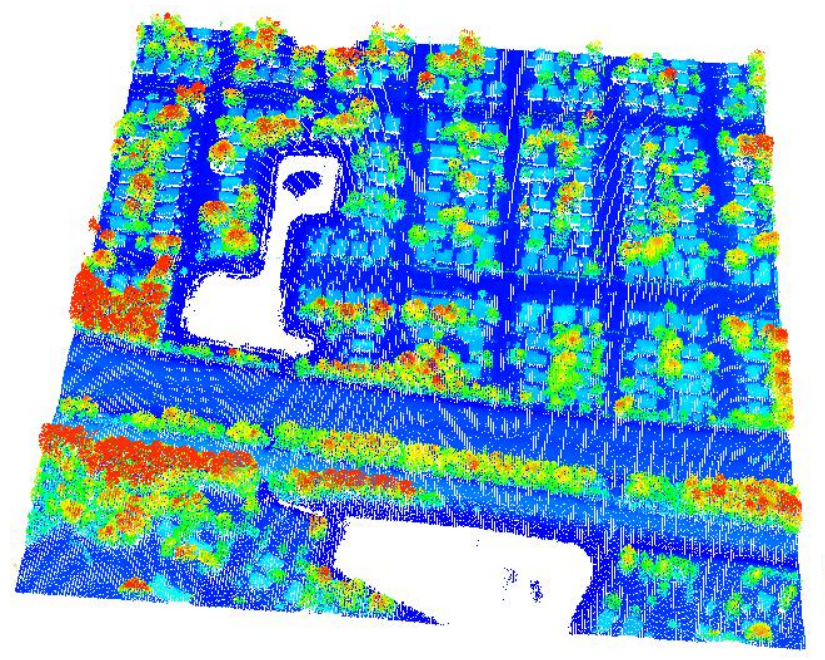

(a)raw data

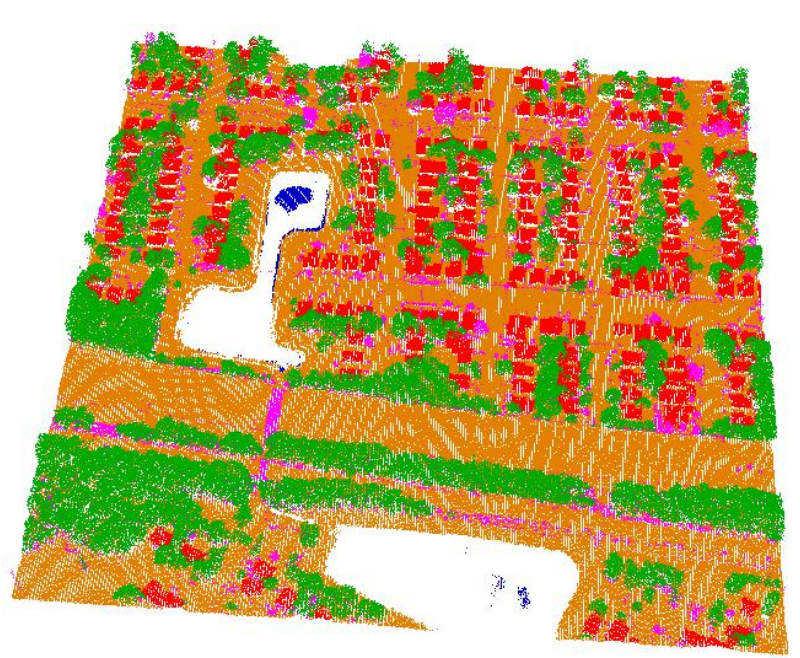

(b)classification results

Figure 3. Results of scene III 
Compared with other methods, the classification accuracy of the method is higher, for example, the recognition result of small objects is significantly better than the other three methods. The classification result of Method II is the worst among the four methods, which means that the classification of points as a basic unit is significantly better than the point-based method. Method I is superior to the identification of small objects in distinguishing large objects, however, it cannot effectively identify features in mixed point clouds. Although Method III has a certain effect on the recognition of features, the accuracy of recognition of small objects needs to be further improved. The method of this paper can achieve better results for the recognition of small objects. This is because the method uses the sparse coding method for dictionary training and feature expression, which can better express the characteristics of spatial entities. The feature of sparse coding combined with LDA to express point sets can obtain more accurate classification results. Figures 1, 2 and 3 visually show the classification results of the method. It can be seen from Figures 1(b), 2(b) and 3(b) that most of the points in this method are correctly classified.

\section{CONCLUSIONS}

This paper proposes a method for extracting the shape features of object objects to classify and identify LiDAR point clouds. In this method, the LiDAR point cloud is segmented into multilevel point sets by using a multi-level point set segmentation method based on an exponential threshold. When segmenting point clouds, ensure that each point set contains the necessary points for extracting the shape features of various objects. The SCLDA model is constructed to extract and encode multi-level point features. Then, the point set is jointly marked by identifying the inheritance of the result by means of point sets at different levels. The SCLDA model we built has certain flexibility and scalability, and can be extended to the classifier of multi-feature channels, thereby further improving the accuracy of point cloud classification. The experimental results show that the proposed method utilizes the correlation between multi-level point sets and effectively captures the salient features of the point set through the SCLDA model. It can classify the point cloud of complex scenes with high classification accuracy, especially for small objects.

The hierarchy of point clouds can be constructed using semantic relationships or visual similarities between categories. In the future, layered learning and multiple feature expressions will be incorporated into the framework of this paper to further improve classification accuracy.

\section{REFERENCES}

[1] Langley P,1994. Selection of relevant features in machine learning. Defense Technical Information Center, 1994.

[2] Guo B., Huang X., Zhang F.,2015. Sohn G. Classification of airborne laser scanning data using JointBoost. ISPRS Journal of photogrammetry and remote sensing, 100: 71-83.

[3] Boykov Y., Veksler O., Zabih R, 2001. Fast approximate energy minimization via graph cuts. IEEE Transactions on Pattern Analysis and Machine Intelligence, 23(11): 1222-1239.

[4] Shi J., Malik J,1997. Normalized cuts and image segmentation. Proceedings of IEEE Computer Society Conference on Computer Vision and Pattern Recognition.
[5] Biosca J. M., Lerma J. L,2008. Unsupervised robust planar segmentation of terrestrial laser scanner point clouds based on fuzzy clustering methods. ISPRS Journal of photogrammetry and remote sensing, 63(1): 84-98.

[6] Barnea S., Filin S,2008. Segmentation of terrestrial laser scanning data by integrating range and image content. The Proceedings of XXIth ISPRS Congress.

[7] Comaniciu D., Meer P,2002. Mean shift: a robust approach toward feature space analysis. IEEE Transactions on Pattern Analysis and Machine Intelligence, 24(5): 603-619.

[8] Barnea S., Filin S,2013. Segmentation of terrestrial laser scanning data using geometry and image information. ISPRS Journal of photogrammetry and remote sensing, 76: 33-48.

[9] Rusu R. B., Marton Z. C., Blodow N., Dolha M., Beetz M,2008. Towards 3D point cloud based object maps for household environments. Robotics and Autonomous Systems, 56(11): 927-941.

[10] Yokoyama H., Date H., Kanai S., Takeda H,2013. Detection and classification of pole-like objects from mobile laser scanning data of urban environments. International Journal of CAD/CAM, 13(2): 31-40.

[11] Weinmann M., Jutzi B., Mallet C,2013. Feature relevance assessment for the semantic interpretation of $3 \mathrm{D}$ point cloud data. ISPRS Annals of the Photogrammetry, Remote Sensing and Spatial Information Sciences, 5: W2.

[12] Weinmann M., Urban S., Hinz S., Jutzi B., Mallet C,2015. Distinctive 2D and 3D features for automated large-scale scene analysis in urban areas. Computers \& Graphics, 49: 47-57.

[13] Wang Z., Zhang L., Fang T., Mathiopoulos P. T., Tong X., Qu H., Xiao Z., Li F., Chen D,2015. A multiscale and hierarchical feature extraction method for terrestrial laser scanning point cloud classification. IEEE Transactions on Geoscience and Remote Sensing, 53(5): 2409-2425.

[14] Russell B. C., Freeman W. T., Efros A. A., Sivic J., Zisserman A,2006. Using multiple segmentations to discover objects and their extent in image collections. Proceedings of the Computer Vision and Pattern Recognition.

[15] Brodu N., Lague D,2012. 3D terrestrial lidar data classification of complex natural scenes using a multi-scale dimensionality criterion: applications in geomorphology. ISPRS Journal of photogrammetry and remote sensing, 68: 121-134.

[16] Pauly M., Keiser R., Gross M,2003. Multi-scale feature extraction on point-sampled surfaces. Computer Graphics Forum, Eurographics issue, 22(3): 3281-289.

[17] Xiong X., Munoz D., Bagnell J. A., Hebert M,2011.3-D scene analysis via sequenced predictions over points and regions[A].IEEE International Conference on proceedings of the Robotics and Automation (ICRA)[C].2011.2609-2616.

[18] Xu S., Oude E. S., Vosselman G,2012. Entities and features for classification of airborne laser scanning data in urban area[A]. The International Archives of the Photogrammetry, Remote Sensing and Spatial Information Sciences[C]. 257-262.

[19] Rau J. Y., Jhan J. P., Hsu Y. C,2015. Analysis of oblique aerial images for land cover and point cloud classification in an urban environment. IEEE Transactions on Geoscience and Remote Sensing, 53(3): 1304-1319.

[20] Zhang Z,Zhang L,Tong X,et al.2016.A Multi-Level Point Cluster-based Discriminative Feature for ALS Point Cloud Classification[J].IEEE Transactions on Geoscience and Remote Sensing,54(6),3309- 3321.

[21] Hartigan J. A., Wong M. A,1979. Algorithm AS 136: A kmeans clustering algorithm. Journal of the Royal Statistical Society (Series C: applied atatistics), 28(1): 100-108.

[22] Zhang Z, Zhang L,Tong X,et al.2016.IEEE Transactions on Geoscience and Remote Sensing,54(12),7309-7322. 